\title{
Téoros
}

Revue de recherche en tourisme

\section{De quelques transformations récentes au sein des administrations nationales du tourisme}

\section{Louis Jolin}

Volume 19, numéro 3, automne 2000

Organisations touristiques en mutation

URI : https://id.erudit.org/iderudit/1071736ar

DOI : https://doi.org/10.7202/1071736ar

Aller au sommaire du numéro

Éditeur(s)

Université du Québec à Montréal

ISSN

0712-8657 (imprimé)

1923-2705 (numérique)

Découvrir la revue

Citer cet article

Jolin, L. (2000). De quelques transformations récentes au sein des administrations nationales du tourisme. Téoros, 19(3), 5-9.

https://doi.org/10.7202/1071736ar d'utilisation que vous pouvez consulter en ligne. 


\section{I : $4: 4:$ Y $: 5:=$}

\section{De QUELQUes tRANSFORMATIONS RÉCENTES AU SEIN DES ADMINISTRATIONS NATIONALES DU TOURISME}

\section{Louis Jolin}

Les États, qui interviennent dans le domaine du tourisme pour de nombreuses raisons, ont revu ces dernières années leur façon de faire et ont mis sur pied des organismes de cogestion avec le secteur privé, reflétant ainsi une volonté de partenariat organique avec lui.

\section{LES RAISONS DE L'INTERVENTION DE L'ÉTAT EN TOURISME}

Le tourisme est un secteur de l'activité économique au sein duquel l'État est particulièrement actif malgré la tendance marquée des dernières années au désengagement et à la déréglementation. Les raisons de la présence de l'État sont multiples. Dans sa thèse de doctorat portant justement sur le rôle de l'État dans le domaine du tourisme, Marion Joppe (1983) affirme ce qui suit :

Pour justifier son intervention dans un domaine qui traditionnellement relève de l'initiative privée, l'État s'appuie sur un certain nombre de caractéristiques inhérentes au phénomène touristique : le dépaysement et le délassement qu'il procure à l'individu ont un effet bienfaisant sur sa santé physique ou psychique, mais contribuent également à améliorer la compréhension entre les peuples et la condition de vie tant des touristes que des hôtes. Le tourisme étant étroitement imbriqué avec le reste de l'économie, il est un outil important pour les politiques en matière de devises, d'emploi, de régionalisation, de répartition de revenus... L'attrait principal du tou- risme étant l'environnement, son développement incite à la protection des ressources touristiques : sites et monuments naturels, historiques, culturels, flore et faune.

Mais le phénomène revêt aussi des caractéristiques négatives qui justifient l'intervention étatique. Le contact entre touristes et hôtes peut entraîner des conflits, en particulier lorsque le rapport touristes/autochtones est très élevé, lorsque le contraste entre les niveaux de vie des deux groupes est trop important ou lorsqu'il y a incompatibilité entre les mours des visiteurs et des visités.

En fait, les raisons de l'intervention de l'État s'articulent autour de deux axes principaux : la contribution du tourisme, sous divers aspects, et la grande vulnérabilité du phénomène et de ses acteurs.

Le tourisme contribue notamment à l'économie nationale (par l'entrée de devises, la création d'emplois, l'apport d'investissements...), au développement socio-économique des régions, à la mise en valeur de l'environnement naturel et culturel des lieux visités, à l'éducation, à l'épanouissement culturel et à la santé physique et psychique des pratiquants. En ce sens, c'est une activité qui mérite d'être encouragée par les États, d'autant plus que la création d'emplois y est relativement facile et peu coûteuse et que la réputation nationale peut bénéficier de la qualité de l'accueil des ressortissants du pays hôte.

Par contre, le tourisme et ses acteurs sont vulnérables et méritent de ce fait une attention particulière de la part de l'État : vulnérabilité du tourisme comme industrie soumise aux aléas du climat, à la situation politique instable de certains pays, aux fluctuations du taux de change, à la saisonnalité des activités et des emplois, à la santé de l'économie en général ; vulnérabilité du touriste lui-même qui achète à distance, sans voir le produit, en temps différé par rapport à sa consommation, qui doit trouver à se loger et à se nourrir dans un environnement étranger ; vulnérabilité enfin des «visités », des communautés d'accueil qui peuvent souffrir d'un envahissement massif des touristes (acculturation des populations, folklorisation des traditions culturelles, destruction de sites naturels et culturels, modifications trop rapides de l'économie locale, etc.). Pour compenser les effets négatifs du tourisme, les Etats interviennent comme régulateur économique pour protéger le touristeconsommateur, ainsi que les richesses naturelles et culturelles des lieux visités.

\section{LES RÔLES ET LES FONCTIONS}

L'intervention de l'État dans le secteur du tourisme est généralement l'affaire de nombreux ministères ou organismes gouvernementaux, notamment ceux qui sont responsables des finances, de la sécurité 
publique, de la protection du consommateur, de l'industrie, de l'environnement, de la culture, du transport..., mais l'un de ceux-ci est généralement responsable de la politique du tourisme et de la concertation interministérielle que le domaine requiert. Pour un tel organisme officiel ou un ministère particulièrement interventionniste, les fonctions seront nombreuses et toucheront diverses dimensions du phénomène touristique. Pour un autre, qui laisse à l'entreprise privée l'essentiel du développement touristique, les fonctions pourront être beaucoup plus restreintes.

L'Organisation mondiale du tourisme a répertorié les diverses fonctions habituellement exercées par les organismes officiels du tourisme. Ces fonctions sont énumérées au tableau 1.

La crise des finances publiques et, plus globalement, la crise existentielle des dernières années, que les États ont vécu dans un contexte de mondialisation de l'économie, les ont amenés à revoir leur mission et leurs diverses interventions aux plans économique, social et culturel. Dans le secteur du tourisme, secteur hautement concurrentiel à l'échelle internationale, les changements ont été manifestes.

\section{DES CHANGEMENTS AU CADRE INSTITUTIONNEL}

Dans un document produit en 1996, le Comité du tourisme de l'OCDE souligne la refonte du cadre institutionnel dans plusieurs pays membres :

Le rôle de l'État et des organismes publics en charge des questions touristiques continue d'être questionné. Comme nous le constaterons au cours des paragraphes suivants, le tourisme est un phénomène complexe qui nécessite une approche pluridisciplinaire afin de mieux comprendre ce qu'il représente. Le rôle de l'État dans le tourisme est de plus en plus souvent jugé sur ses capacités à fournir aux autres niveaux de

\section{TABIEAU 1}

Pincipales fonctions tes organismes officiels dil tourisme

- Représentation officielle gouvernementale en matière touristique

- participation aux organismes internationaux

- négociation des accords bilatéraux ou multilatéraux

- Recherches, études et enquêtes statistiques

- Promotion du tourisme

- à l'étranger

- à l'intérieur du pays (bureaux d'information, campagnes publicitaires)

- Planification et développement du tourisme

- Réglementation et contrôle des entreprises

- Facilitation des formalités d'entrée et de sortie pour les étrangers

- Accueil et information (bureaux d'information et d'accueil, services de guides et d'interprètes...)

- Formation professionnelle

- Préservation, protection et utilisation des ressources touristiques et culturelles (aménagement des parcs, des réserves, protection du patrimoine)

Source : OMT, Buts, rôles et fonction des organismes officiels du tourisme

gouvernement mais aussi et surtout à l'industrie les informations dont ils ont besoin pour élaborer leurs plans stratégiques d'action en matière d'investissement, de promotion et de communication.

Toutefois, dans un climat économique incertain, les contraintes imposées sur les ressources des gouvernements ont poussé les administrations du tourisme à ajuster l'utilisation des fonds publics dans le domaine du tourisme et à affiner leurs stratégies politiques. Elles ont aussi rendu souhaitable la coordination et le partage des responsabilités avec les autres autorités publiques ainsi que le développement de partenariats avec le secteur privé, notamment dans le domaine de la promotion. Cela implique parfois une refonte complète du cadre institutionnel existant (Comité du tourisme de l'OCDE, 1996: 19).

Le document énumère quelques axes et quelques exemples de cette refonte :

- Une coordination interministérielle plus accentuée que par le passé (notamment, en Espagne, en Finlande et en Norvège ; l'Italie a supprimé le ministère du Tourisme «pour en faire une structure à vocation multidisciplinaire placée sous la compétence du Premier ministre »

- Une coopération avec les régions et les autorités locales : des pays ont mis en place des comités pour exprimer en termes politiques les propositions venant des régions et des collectivités locales (la Hongrie, l'Espagne, la Grèce, l'Italie, entre autres).

- La mise en place de structures de discussions ou de partenariat avec le secteur privé : on crée des unités ou des organes, sur la base d'une nouvelle relation de travail entre les partenaires publics et le secteur privé, dans le domaine de la promotion et du marketing (à titre d'exemples, la Commission canadienne du tourisme et Maison de la France expérimentent des formes de cogestion; plus modestement, des organes de consultation et d'orientation sont composés de représentants du secteur privé en Australie, au Danemark, en Finlande...). 
Plus globalement, on constate une tendance générale à la décentralisation et à la privatisation qui ne remet pas en cause une nécessaire coordination et une intégration des interventions à la politique nationale. S'il y a toujours place pour un organisme officiel du tourisme (ministère, office national du tourisme ou direction du tourisme au sein d'un ministère plus large), on délègue dorénavant certaines fonctions à des organismes publics ou mixtes, voire à des organisations privées. La reconnaissance du tourisme comme industrie, comme phénomène économique de première importance, avait amené plusieurs États à se doter d'un organisme officiel fort ou d'un ministère, dans le plein sens du terme, en charge de tous les aspects du tourisme, y compris même, assez fréquemment, celui de la formation des ressources humaines. (Jusqu'à il y a une dizaine d'années, l'Institut de tourisme et d'hôtellerie du Québec était un service du ministère québécois du Tourisme; il y a toujours une direction de la formation professionnelle au ministère du Tourisme du Maroc). La crise de l'État, dans ses divers rapports avec la société comme instituteur du social, régulateur économique, responsable de 1'ordre intérieur et de la sécurité nationale, réducteur d'incertitudes (Rosanvallon, 1984), a cependant bouleversé l'approche et la façon de faire.

\section{L'ÉTAT OPTIMAL EN TOURISME?}

Dans son texte Le Canada des années 90 : effondrement ou renaissance, Kimon Valaskakis (1990: 171) tente de se situer entre l'État maximal (en crise) et l'État minimal (un leurre). Entre la « boulimie » et l' « anorexie nerveuse », il propose un État optimal, selon trois idées porteuses de changement: «d'abord un secteur public 'productif', ensuite un secteur privé à 'responsabilité sociale' et enfin une relation organique entre les deux, nécessaire à la croissance des deux secteurs ». Ces idées ont semblé guider la refonte du cadre institutionnel en tourisme au Canada, au Québec et ailleurs dans le monde. Regardons-y de plus près.

Des gouvernements distinguent de plus en plus les fonctions pour une meilleure productivité : dans le secteur de la promotion et du marketing touristiques, il importe d'être efficace, de prendre des décisions rapides selon la conjoncture et d'augmenter la force de frappe en coordonnant les efforts du public et du privé. On élabore des indicateurs de performance précis et on emploie des méthodes de gestion qui se rapprochent de ce qui a cours dans le secteur privé. L'entente de gestion qui consacre Tourisme Québec comme unité autonome de gestion, au sein du ministère de l'Industrie, du Commerce, de la Science et de la technologie, comprend l'énumération de tels indicateurs et accorde une souplesse administrative qu' on ne trouve habituellement pas dans la fonction publique.

Le secteur privé est aussi invité à se responsabiliser davantage dans le domaine social, tant au niveau de la formation des ressources humaines en tourisme (formation continue des employés, stages en entreprises) que dans la poursuite d'objectifs de développement durable dans le secteur du tourisme. "La qualité en matière de tourisme devient un élément déterminant dans une stratégie politique du tourisme» (Comité du tourisme de l'OCDE, 1996 : 21). Le développement de la qualité touche à la fois 1'amélioration de l'offre, la formation du personnel et un meilleur étalement dans le temps des flux touristiques.

Plusieurs pays ont pris le virage de la qualité (Canada, Autriche, Espagne, Grèce, Luxembourg, Portugal, Turquie...).

Enfin, on met sur pied de nouveaux organismes publics ou mixtes pour réaliser certaines fonctions (promotion, formation, développement...) : ils comptent des représentants des secteurs public et privé ou s'appuient sur la participation conjointe des acteurs publics et privés, tant en ce qui concerne les décisions que le financement (CCT, Maison de la France, Bureau du tourisme néerlandais, etc.). Cette délégation fonctionnelle des pouvoirs à des organismes relativement autonomes - dans bien des cas, le ministre responsable ou le conseil des ministres continue néanmoins à nommer les membres du conseil d'administration - traduit une volonté de créer un partenariat organique dans une démarche de cogestion.

\section{DES EXEMPLES DE PARTENARIAT ORGANIQUE}

La nouvelle Loi sur la Commission canadienne du tourisme, sanctionnée en octobre dernier, confirme l'orientation qui a présidé

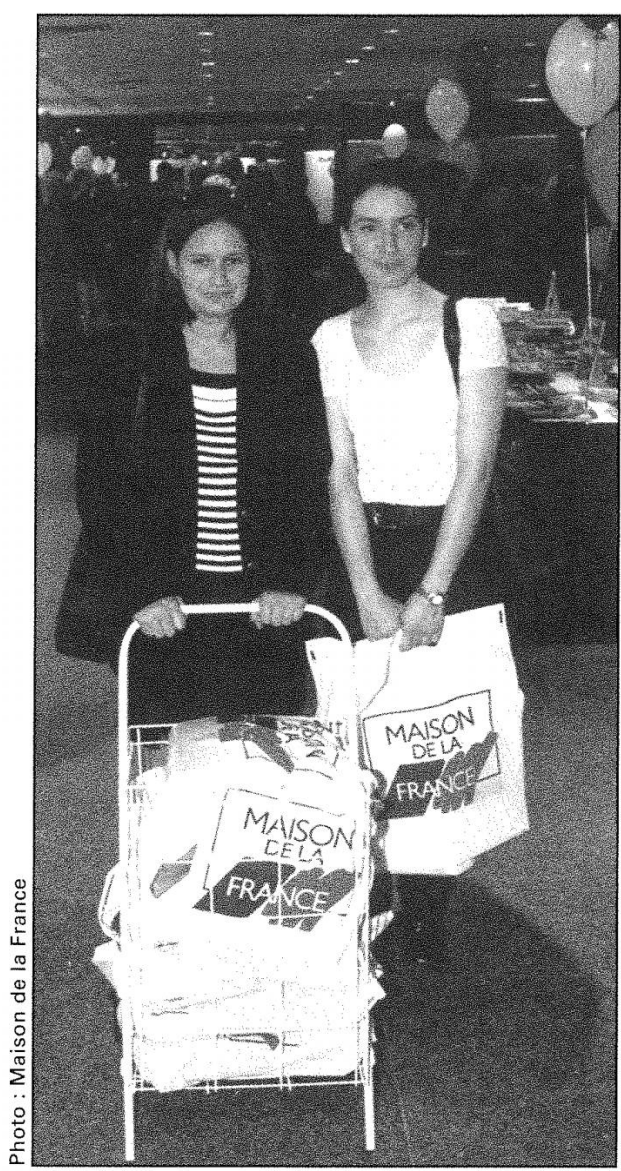

à la création de la CCT en $1995^{1}$ : le conseil d'administration qui assure « la conduite des affaires et des activités de la Commission » (article 7) est composé d'au plus vingt-six administrateurs dont seize proviennent du secteur privé (article 11). Outre le sous-ministre de l'Industrie qui est administrateur d'office (article 13), les administrateurs publics sont nommés parmi les personnes désignées par les ministres provinciaux ou territoriaux chargés du tourisme, personnes qui sont des sous-ministres ou leur équivalent ou des dirigeants d'organisme provincial ou territorial (article 12). Par diverses dispositions, la loi se soucie que les administrateurs proviennent des diverses provinces ou territoires du Canada (article 11). Tous les administrateurs sont nommés en dernier lieu par le ministre de l'Industrie, avec l'agrément du Conseil des ministres au fédéral (articles 11 et 12). La Commission a ses propres employés dont plusieurs proviennent du ministère de l'Industrie qui les a préalablement licenciés selon la Loi sur la gestion des finances publiques (article 29). Le budget de base provient du gouvernement fédéral, mais les provinces ou les entreprises sont invitées à s'associer aux opérations de marketing, de dé- 


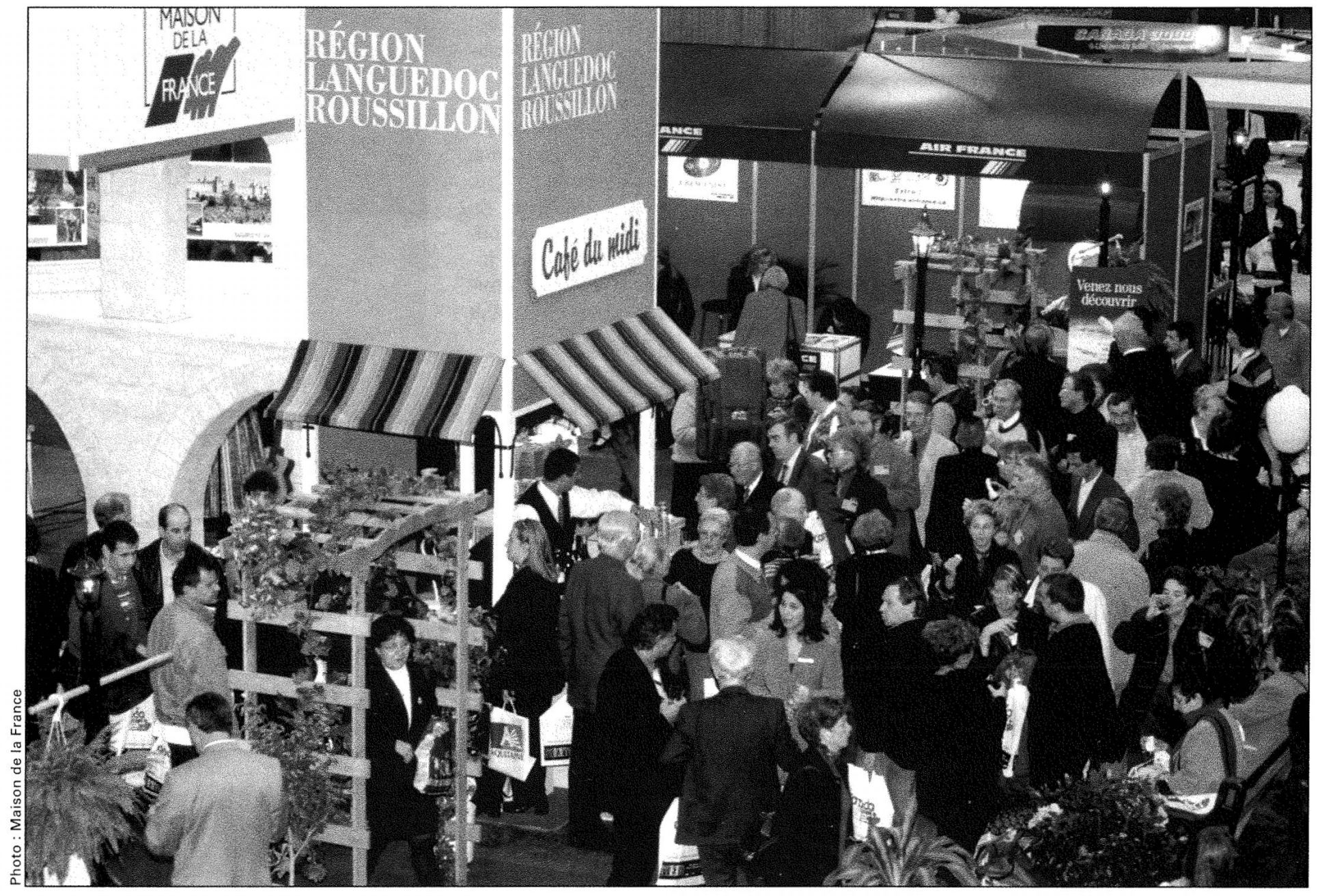

veloppement de produits, de services organisationnels, de recherche et de diffusion externe. Le budget de la CCT pour 2000-2001 consiste « en un fonds de programme du gouvernement s'élevant à 56,1 millions de dollars, d'une somme de 71,6 millions de dollars en espèces ou services rendus à titre de contribution des partenaires, ainsi que d'un montrant additionnel de 11,3 millions de dollars au chapitre des salaires $^{2} \gg$.

Maison de la France (MDLF) va encore plus loin dans la volonté de constituer un partenariat organique. Juridiquement, il s'agit d'un groupement d'intérêt économique (GIE) sous l'autorité du ministre chargé du tourisme. Les adhérents, environ 1000 , proviennent de tous les secteurs intéressés par le tourisme (collectivités territoriales, offices de tourisme, personnes physiques et morales des secteurs de la restauration, de l'hébergement, du transport, du voyage, autres fournisseurs de services aux touristes, etc.). L'assemblée des membres comme telle est constituée de 70 personnes qui proviennent princi- palement de trois collèges qui regroupent les adhérents (à l'exception du quatrième collège, celui des membres associés). Le conseil d'administration se compose de vingt-sept personnes :

- quatorze administrateurs permanents, dont sept représentants de l'État, cinq présidents des grandes associations sectorielles et deux représentants choisis dans le secteur de l'hôtellerie ;

- treize administrateurs renouvelables, dont neuf sont nommés par le ministre chargé du tourisme, principalement au sein des entreprises touristiques présentes sur les marchés internationaux, trois sont élus par chacun des trois premiers collèges de l'assemblée générale et un membre d'honneur (le président sortant).

Comme on peut le constater, par rapport à la Commission canadienne du tourisme, la représentation du secteur privé ne dépend pas uniquement de la décision du ministre (certains administrateurs y sont de façon statutaire, d'autres sont élus par les mem- bres) et le budget de base est constitué en partie de la contribution financière des adhérents. En 1998, les revenus totaux de MLDF ont été de l'ordre 326837000 francs français : 165150000 francs provenaient des partenaires de MLDF (51\%) et 161687000 de l'État (49\%).

Un autre exemple est celui du Conseil du tourisme des Pays-Bas (NBT). Unité semipublique autonome, le NBT vise à promouvoir le tourisme aux Pays-Bas, tant à l'échelle nationale qu'internationale, grâce à des efforts concertés entre les différents intervenants du secteur. Le NBT compte quatre types de partenaires : les partenaires associés (ministère des Affaires économiques, KLM, associations sectorielles); l'industrie touristique (entreprises) ; les offices d'information touristique engagés dans des activités promotionnelles; les entreprises ne provenant pas de l'industrie (partenaires potentiels). Il n'y a pas d'assemblée générale comme telle, mais un conseil d'administration composé de cinq partenaires invités par le président du conseil, un comité consultatif composé de 
treize partenaires et une équipe d'employés regroupés au sein de six départements. Le NBT est financé par les secteurs public et privé dans une proportion à peu près égale. Le plan marketing stratégique pour 19971999 prévoyait un budget de 75 millions de florins, dont 36 millions de l'industrie et 39 millions du ministère des Affaires économiques ${ }^{3}$ ).

\section{DE QUELQUES LIMITES À LA REFONTE DU CADRE INSTITUTIONNEL}

Un rapide survol de la situation dans différents pays permet de faire ressortir deux limites à la refonte du cadre institutionnel : peu de pays ont carrément opté pour la privatisation et les organes de cogestion, auxquels sont délégués des pouvoirs étatiques, n'ont généralement que des missions spécifiques.

Il y a quelques années, la Suède avait tenté une expérience de privatisation totale dans le domaine du marketing et de la commercialisation ; or, elle a dû revenir en arrière parce que «les entreprises n'étaient pas en mesure de promouvoir l'image d'un pays mais qu'elles privilégiaient généralement leurs intérêts particuliers au détriment de l'intérêt général » (Comité du tourisme de l'OCDE, $1996: 20$ ).

Par ailleurs, toutes les fonctions de l'État ne peuvent être déléguées à un organisme de cogestion avec le secteur privé. Le Québec a failli emprunter cette voie avec la Loi sur la Société du tourisme au Québec, adoptée en 1994 par l'Assemblée nationale, à la veille des élections, mais qui n'a jamais été mise en vigueur par le nouveau gouvernement, celui du Parti québécois. Cette loi instituait un organisme public chapeauté par un conseil d'administration composé de représentants du secteur privé, « nommés par le gouvernement, sur proposition du ministre, après consultation d'organismes qu'il considère représentatifs des milieux concernés par les activités de la Société » (article 14). Les fonctions déléguées à la Société par la Loi couvraient presque tout le registre d'intervention d'un ministère responsable du tourisme : de la promotion et de la commercialisation à l'aide financière aux entreprises, de la diffusion de l'information touristique à la concertation générale des intervenants privés et publics. L'article 2 de la Loi se lisait comme suit :
La Société a pour objet de développer et de soutenir l'industrie touristique du Québec.

À cette fin, la Société identifie les besoins en développement, définit et met en ouvre des orientations, des plans d'action et des stratégies d'intervention et établit et gère des programmes, dans le cadre de la politique gouvernementale en tourisme.

Même si la Société restait sous la tutelle du gouvernement, l'ampleur de la délégation a suscité des réserves et des inquiétudes ; de plus, les employés de la nouvelle Société, provenant pour une large part du ministère de l'Industrie, du Commerce, de la Science et de la Technologie, n'auraient plus été soumis à la Loi de la fonction publique après le transfert et l'expiration des conventions collectives en vigueur au moment du transfert. Pour toutes ces raisons, le nouveau gouvernement de l'époque a reculé et a choisi de faire de Tourisme Québec une simple unité autonome de service qui, malgré les intentions de partenariat avec le privé, n'est pas un organisme distinct de cogestion. La Société n'aurait-elle pas eu davantage d'avenir si l'on avait restreint sa mission à la promotion et à la commercialisation ? La Commission canadienne du tourisme semble prima facie assumer l'ensemble des fonctions de l'État canadien, mais rappelons que l'essentiel des pouvoirs en tourisme demeure du ressort des provinces et que le champ de compétences du fédéral dans le secteur du tourisme (outre son traditionnel pouvoir de dépenser) est justement la promotion et la commercialisation sur les marchés étrangers. Même le British Tourist Authority (BTA) - qui réunit sous son autorité les offices nationaux du tourisme (Écosse, Pays de Galles, Angleterre) et le secteur privé -, qui a servi d'exemple au projet de Société du tourisme du Québec, a pour mandat de développer et de promouvoir une image de marque pour l'industrie britannique (Hollier, 1995 : 39-40).

Les administrations nationales du tourisme ont connu des transformations importantes dans le sens d'une délégation à un organisme de cogestion des fonctions de promotion et de commercialisation, mais les États semblent toujours vouloir assumer, pour le moment du moins, certaines fonctions de façon centralisée. Les fonctions de planification et de réglementation sont généralement sous la responsabilité directe du gouvernement qui reste le maître de la politique du tourisme.

Louis Jolin est professeur au Département d'études urbaines et touristiques de l'UQAM et membre du comité de rédaction de Téoros.

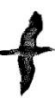

\section{NOTES}

1 À sa création, la Commission canadienne du tourisme a été constituée par le décret C.P. 1995-110 du 31 janvier 1995 qui faisait suite à une décision initiale prise par le Conseil du trésor. La nouvelle Commission sera régie par les dispositions de la Loi sur la Commission canadienne du tourisme (Projet de loi C-5, $2^{\mathrm{e}}$ session, $36^{\mathrm{e}}$ législature, 48-49 Elisabeth, 1999-2000), sanctionnée, mais dont l'entrée en vigueur est prévue vraisemblablement pour le début de 2001 (à une date fixée par décret).

2 Commission canadienne du tourisme, Plan d'affaire de la CCT, 2000-2001 - Sommaire exécutif.

3 NBT, Stategic Marketing Plan, 1997-1999.

\section{BIBLIOGRAPHIE}

Comité du tourisme de l'OCDE (1996), Politique du tourisme et tourisme international dans les pays de l'OCDE 1993-1994, Paris, OCDE.

Hollier, Robert (1995), The National and Regional Structures on Official Tourist Organisations in European Countries, Paris, Conseil national du tourisme, janvier, p. 39-40.

Joppe, Marion (1983), L'intervention de l'État en tourisme, thèse de doctorat, Université de droit, d'économie et de sciences d'Aix-Marseille.

Rosanvallon, Pierre (1984), La crise de l'Étatprovidence, Paris, (Seuil), $190 \mathrm{p}$.

Valaskakis, Kimon (1990), Le Canada des années 90 : effondrement ou renaissance, Montréal, Transcontinental. 